Stéphanie BLOT-MACCAGNAN, Procédure criminelle et défense de l'accusé à la fin de l'Ancien Régime. Étude de la pratique angevine

\title{
Sébastien Annen
}

\section{(2) OpenEdition}

Journals

\section{Édition électronique}

URL : https://journals.openedition.org/ahrf/12165

DOI : 10.4000/ahrf.12165

ISSN : 1952-403X

Éditeur :

Armand Colin, Société des études robespierristes

\section{Édition imprimée}

Date de publication : 1 septembre 2011

Pagination : 207-208

ISBN : 978-2-200-92700-4

ISSN : 0003-4436

Référence électronique

Sébastien Annen, «Stéphanie blot-maccagnan, Procédure criminelle et défense de l'accusé à la fin de l'Ancien Régime. Étude de la pratique angevine ", Annales historiques de la Révolution française [En ligne], 365 | Juillet-septembre 2011, mis en ligne le 13 décembre 2011, consulté le 23 avril 2022. URL : http:// journals.openedition.org/ahrf/12165; DOI : https://doi.org/10.4000/ahrf.12165

Ce document a été généré automatiquement le 23 avril 2022.

Tous droits réservés 


\title{
Stéphanie BLOT-MACCAGNAN, Procédure criminelle et défense de l'accusé à la fin de l'Ancien Régime. Étude de la pratique angevine
}

\author{
Sébastien Annen
}

\section{RÉFÉRENCE}

Stéphanie BLOT-MACCAGNAN, Procédure criminelle et défense de l'accusé à la fin de l'Ancien Régime. Étude de la pratique angevine, Rennes, Presses universitaires de Rennes, 2010, 324 p., ISBN 978-2-7535-1058-6, $20 €$

1 La thèse qu'a soutenue Stéphanie Blot-Maccagnan en 2002, sous la direction de JeanMarie Carbasse et de Sylvain Soleil, intéressera moins directement les historiens de la Révolution que les dix-huitièmistes soucieux de mieux comprendre le droit et la pratique judiciaire avant 1789. Elle s'inscrit largement dans la lignée des travaux d'Antoine Astaing qui, déjà en 1999, interrogeaient la place réservée à la défense de l'accusé dans la doctrine pénale d'Ancien Régime. Pour approfondir la réflexion, Stéphanie Blot-Maccagnan cherche à valoriser les fonds judiciaires de trois sénéchaussées d'Anjou (Angers, Beaufort-en-Vallée et Saumur) et les mobilise pour confirmer, infirmer ou nuancer l'application stricte du droit dictée par l'ordonnance criminelle de 1670 et les ouvrages de doctrine. Après une partie qui s'attache à présenter de façon générale les deux modes de résolution des conflits (résolution amiable et/ou recours à la justice publique), la thèse suit pas à pas la procédure criminelle, de l'instruction (préparatoire et définitive) au jugement, lui-même complété par les voies de recours (appel et grâce).

2 Si le formalisme de la procédure reste le premier moyen de défense de l'accusé (quelle que soit l'époque), d'autres moyens s'offrent à ce dernier. Il peut par exemple formuler 
une plainte réciproque contre le plaignant et renverser ainsi l'accusation. Il peut nier les faits en bloc, surtout sous la torture : ses aveux, renforcés par des témoignages à charge, viendraient automatiquement le condamner dans le cadre du système des preuves légales. Il peut reprocher des témoins, présenter des faits justificatifs, des exemptions péremptoires qui pourront arrêter ou annuler l'action judiciaire. Il peut aussi - mais le fait rarement - récuser ses juges ou les prendre à partie, faire appel de la sentence auprès du parlement compétent (ici, le parlement de Paris) ou, le cas échéant, demander grâce.

Ce livre constitue assurément un remarquable manuel sur la procédure criminelle d'Ancien Régime et l'on conseillera vivement l'organigramme de ladite procédure, situé en annexe (p. 299-300). Mais au terme d'une réflexion qui ne fait que reprendre dans l'ensemble ce que les bons manuels d'histoire du droit nous apprennent déjà, on ne peut que regretter les partis pris et les insuffisances de ce travail.

4 Pour faire refluer «l'historiographie dominante qui, depuis la Révolution, reprend l'opinion des philosophes et réformateurs du XvIII siècle, notamment Montesquieu, Beccaria et Voltaire » (p. 15), Stéphanie Blot-Maccagnan cherche à démontrer que la loi et la doctrine reconnaissent la défense de l'accusé comme un droit naturel et lui proposent des moyens de défense. Au point que : « ce système procédural, lorsqu'il est bien appliqué, est particulièrement favorable aux accusés puisqu'il leur permet d'échapper à la justice, et ce, quelle que soit l'intime conviction des juges » (p. 15). Une meilleure prise en compte de l'évolution de la pratique judiciaire en ce domaine aurait sans doute permis de nuancer un propos parfois trop confiant. Si les quelques archives analysées mettent en valeur différentes stratégies de défense adoptées par les accusés, elles ne permettent certes pas d'affirmer une thèse de portée générale qui, poussée à son extrémité, rend les accusés dits passifs responsables de leur propre malheur. Après tout, les moyens de défense ne sont que «de simples moyens mis à leur disposition pour se défendre. S'ils veulent une sentence plus clémente, il leur appartient de les utiliser» (p. 294). L'auteur loue en conséquence la combativité et l'ingéniosité des accusés ainsi que la déontologie des juges qui garantissent la légalité de la procédure et, conformément à la doctrine, se font les premiers protecteurs des accusés.

On notera cependant une contradiction non résolue. Stéphanie Blot-Maccagnan insiste en effet et à juste titre sur le « rôle pondérateur » (p. 298) de la pratique judiciaire et de ces juges qui savent tempérer les rigueurs de la loi. "Il est certain, dit-elle, que les accusés reçoivent le conseil d'un avocat, malgré l'interdiction légale» (p. 123), sans quoi l'on ne comprendrait pas que l'accusé parvienne à soulever les nullités de la procédure et à élaborer de vraies stratégies de défense par une connaissance précise du système des preuves légales. Mais quand la pratique vient modérer et contourner à ce point les règles de droit, c'est bien le signe que la procédure, telle qu'elle aurait dû être respectée, ne convient pas. Si elle admet que l'accusé puisse se défendre, elle est donc cependant loin de lui faciliter la tâche.

6 Au reste, Stéphanie Blot-Maccagnan atténue elle-même son propos en conclusion lorsqu'elle rappelle l'atrocité des peines, le maintien (dans les textes mais exceptionnellement dans la pratique) de la question préparatoire (jusqu'en 1780) et préalable (jusqu'en 1788), l'impossibilité de présenter des faits justificatifs avant la visite $\mathrm{du}$ procès et l'absence d'avocat reconnu lorsque le procès suit la voie extraordinaire, qui sont «autant de travers graves qui justifient à eux seuls les critiques» (p. 297). Il est donc dommage qu'une vision partielle de l'historiographie, 
qui depuis quelque temps déjà, a cessé d'explorer la justice d'Ancien Régime uniquement à l'aune du corpus voltairien, ait conduit l'auteur à un mouvement de balancier inverse aussi radical. Rappelons que la logique interne d'un système judiciaire pluriséculaire peut s'analyser sans nier ni les moyens de défense, ni les limites d'une justice qui, tout en fonctionnant au quotidien, n'en était pas moins l'objet de critiques de la part des contemporains, qu'ils soient ou non juristes. Le croisement judicieux des travaux des historiens et des historiens du droit permet heureusement de dépasser ce débat sans fin qui divise les chercheurs en défenseurs et en contempteurs de la justice d'Ancien Régime. 